\title{
Low-energy excitations in strongly correlated materials: A theoretical and experimental study of the dynamic structure factor in $\mathrm{V}_{2} \mathrm{O}_{3}$
}

\author{
Federico Iori, ${ }^{1,2, *}$ Fanny Rodolakis,,${ }^{3,4, \dagger}$ Matteo Gatti, ${ }^{2,5}$ Lucia Reining,,${ }^{1,2}$ M. Upton, ${ }^{6}$ Y. Shvyd'ko, ${ }^{6}$ \\ Jean-Pascal Rueff, ${ }^{4,7}$ and Marino Marsi ${ }^{3}$ \\ ${ }^{1}$ Laboratoire des Solides Irradiés, Ecole Polytechnique, CNRS, CEA-DSM, F-91128 Palaiseau, France \\ ${ }^{2}$ European Theoretical Spectroscopy Facility (ETSF) \\ ${ }^{3}$ Laboratoire de Physique des Solides, CNRS-UMR 8502, Université Paris-Sud, F-91405 Orsay, France \\ ${ }^{4}$ Synchrotron SOLEIL, L'Orme des Merisiers, Saint-Aubin, BP 48, F-91192 Gif-sur-Yvette, France \\ ${ }^{5}$ Nano-Bio Spectroscopy Group, Departamento Física de Materiales, Universidad del País Vasco, Centro de Física de Materiales \\ CSIC-UPV/EHU-MPC and DIPC, Avenida Tolosa 72, E-20018 San Sebastián, Spain \\ ${ }^{6}$ Advanced Photon Source, Argonne National Laboratory, Argonne, Illinois 60439, USA \\ ${ }^{7}$ Laboratoire de Chimie Physique-Matière et Rayonnement, CNRS-UMR 7614, Université Pierre et Marie Curie, F-75005 Paris, France
}

(Received 14 June 2012; published 30 November 2012)

\begin{abstract}
This work contains an experimental and theoretical study of the dynamic structure factor at large momentum transfer $|Q| \sim 4 \AA^{-1}$ of the strongly correlated transition-metal oxide $\mathrm{V}_{2} \mathrm{O}_{3}$. We focus in particular on the transitions between $d$ states that give rise to the spectra below $6 \mathrm{eV}$. We show that the main peak in this energy range is mainly due to $t_{2 g} \rightarrow e_{g}^{\sigma}$ transitions, and that it carries a signature of the phase transition between the paramagnetic insulator and the paramagnetic metal that can already be understood from the joint density of states calculated at the level of the static local density approximation. Instead, in order to obtain theoretical spectra that are overall similar to the measured ones, we have to go beyond the static approximation and include at least crystal local field effects. The latter turn out to be crucial in order to eliminate a spurious peak and hence allow a safe comparison between theory and experiment, including an analysis of the strong anisotropy of the spectra.
\end{abstract}

DOI: 10.1103/PhysRevB.86.205132

PACS number(s): 71.45.Gm, 71.15.-m, 71.30.+h

\section{INTRODUCTION}

The spectroscopy of correlated materials poses continuous challenge to both experimentalists and theoreticians. On one side, correlation goes hand in hand with a strong dependence of materials' properties on parameters such as pressure, temperature, or doping. This can lead to multiple minima in a potential energy surface, phase transitions, and the formation of domains, which can make well-defined experiments difficult and question the reliability of calculations. At the same time, this sensibility constitutes promise for applications where a big response of materials to a small perturbation is desired, such as for intelligent windows or sensors. Metal-insulator transitions are therefore one of the topics at the heart of current condensed matter research. ${ }^{1,2}$ In view of this challenge, a combined experimental and theoretical effort seems to be a promising strategy in order to add a piece of clarification to the puzzle of open questions.

Vanadium sesquioxide $\mathrm{V}_{2} \mathrm{O}_{3}$ is a prototypical correlated material, in which temperature, $\mathrm{Cr}$ doping, or pressure induce a metal-insulator transition ${ }^{3}$ (MIT) between a paramagnetic metal (PM) and a paramagnetic insulator (PI) phase. The PM and PI phases share the same rhombohedral (corundum) crystal structure (which is expanded in the PI phase upon $\mathrm{Cr}$ doping). At low temperature, a monoclinic antiferromagnetic insulator is also formed. For a long time, $\mathrm{V}_{2} \mathrm{O}_{3}$ has been described in terms of a single-band Hubbard model. ${ }^{4}$ However, it is now well recognized that this model is a considerable oversimplification of the complexity of its electronic structure. For instance, an admixture of distinct V $3 d$ components (differently hybridized with $\mathrm{O} 2 p$ states) close to the Fermi level has been evidenced by $\mathrm{x}$-ray-absorption (XAS) measurements. ${ }^{5}$ Moreover, contrary to the common assumption, recently it has been shown that the transition to the metallic phase under pressure is different from the one obtained by changing temperature or underdoping. ${ }^{6-8}$ The combination of photoemission spectroscopy (PES) and manybody calculations (see, e.g., Refs. 9-13) has much contributed to elucidate the valence electronic properties across the phase diagram, and to discriminate between the bulk and the surface contributions. ${ }^{14,15}$ However, PES is intrinsically limited to occupied states and, for example, cannot clarify the evolution across the MIT of the whole V $3 d$ manifold which is spread across the Fermi level (or the band gap in the PI phase).

The aim of this work is to focus on the excitations within the $3 d$ bands in transition-metal oxides, at the example of $\mathrm{V}_{2} \mathrm{O}_{3}$. These weak low-energy excitations are dipole forbidden unless the bands are hybridized, and are therefore not accessible with optical experiments. Instead, they can be studied with inelastic $\mathrm{x}$-ray scattering (IXS), which in its resonant (RIXS) and nonresonant (NIXS) versions is a powerful probe for electronic excitations in condensed matter. Here we concentrate on NIXS. It gives access to the dynamic structure factor $S(\mathbf{Q}, \omega)$. Through the fluctuation-dissipation theorem this quantity is proportional to the imaginary part of the Fourier transform of the density-density response function. This allows one to express $S$ as

$$
S(\mathbf{Q}, \omega)=-\frac{\hbar^{2} Q^{2}}{4 \pi^{2} e^{2} n} \operatorname{Im} \frac{1}{\epsilon_{M}(\mathbf{Q}, \omega)},
$$

where $\mathbf{Q}$ is the transferred momentum, $n$ is the average electron density, and

$$
\epsilon_{M}(\mathbf{Q}, \omega)=\frac{1}{\epsilon^{-1}(\mathbf{Q}, \mathbf{Q}, \omega)}
$$


is inversely proportional to a diagonal element of the inverse dielectric matrix. ${ }^{16}$ For vanishing $\mathbf{Q},(2)$ yields the macroscopic dielectric function that determines optical spectra. For finite $\mathbf{Q}$ the dynamic structure factor (1) contains the longitudinal linear response of the system to an external potential. It gives hence access to collective excitation such as plasmons, and screened electron-hole excitations.

The collective nature of the excitations, or, expressed in terms of mathematics, the mixing of many independentparticle transitions that contribute to a given observed structure in the spectrum, makes the interpretation of experiments complicated. This mixing of transitions has a twofold origin. First, $\epsilon$ is a matrix in reciprocal space, but even in a diagonal approximation the combination of (1) and (2) contains an inversion, which is responsible for the creation of the plasmonic structures in the spectrum. Plasmon excitations in fact are related to zeros of $\operatorname{Re} \epsilon_{M}$, which can be hence identified as peaks of $-\operatorname{Im} 1 / \epsilon_{M}$. Second, when the matrix nature of $\epsilon$ with its off-diagonal elements is fully taken into account, an additional mixing occurs through the matrix inversion. The off-diagonal elements reflect the inhomogeneity of the system; in other words, they are responsible for the crystal local field effects (LFE). These effects are often important for large $\mathbf{Q}$.

IXS experiments performed at large momentum transfer are hence a route of choice to access the $d$ - $d$ transitions, and at the same time theoretical support is highly desirable in order to interpret the measured structures. Here we concentrate on IXS spectra of $\mathrm{V}_{2} \mathrm{O}_{3}$ with significantly large momentum transfer of $\sim 4 \AA^{-1}$, for both the insulating and the metallic phase. The signature of $d$ - $d$ transitions in the dynamical structure factor of transition metals and their oxides has already been investigated in previous studies in the literature. ${ }^{17-22}$ Important insight was obtained, in particular concerning the weight of the LFE ${ }^{18,23,24}$ and the observation of strongly bound excitons. ${ }^{19,20}$ However, a reliable theoretical description of the observations turned out to be very difficult, because a theoretical framework seems to be still missing that is at the same time efficient enough to deal $a b$ initio with this class of materials, and that is able to treat many-body effects on a sufficiently high and consistent level to be applicable to strongly correlated materials.

Indeed, today the most prominent approach to study correlated oxides is dynamical mean field theory (DMFT). ${ }^{25}$ Although based on a model Hamiltonian and therefore dependent on some choices (localized orbitals, effective interaction), it has proven its success in describing and predicting properties based on the one-particle Green's function $G$, in particular photoemission spectra. It is probably the only method today that obtains a metal-insulator transition in paramagnetic $\mathrm{V}_{2} \mathrm{O}_{3},{ }^{9-12}$ all other approaches such as the prominent $\mathrm{GW}$ approximation $^{26}$ of many-body perturbation theory yielding always a metal. However, IXS requires in principle the calculation of a two-particle Green's function $G_{2}$, which contains at least an electron and a hole. These two particles are not independent but subject to an effective interaction due to the induced potentials that are self-consistently created by the excitation. There are a few DMFT calculations for optical properties that are based on $G_{2}$, but usually the electron-hole interaction is completely neglected and absorption is simply calculated from the product $G G,{ }^{4,27}$ neglecting the electronhole attraction and also the LFE. In a metal this may be justified because of screening, but the approximation becomes problematic in the insulating phase. Recent attempts exist to include vertex corrections in optical spectra within DMFT, ${ }^{28}$ but to our knowledge the methods have not been applied to complex materials such as $\mathrm{V}_{2} \mathrm{O}_{3}$, and they have not been tested for finite momentum transfer.

Methods that have been developed for weakly correlated systems such as many-body perturbation theory ${ }^{29}$ or timedependent density functional theory (TDDFT) ${ }^{30}$ do at first sight not seem to be appropriate to describe materials such as $\mathrm{V}_{2} \mathrm{O}_{3}$, but they have the advantage that research concerning neutral excitations is already in an advanced stage-e.g., with many developments concerning the description of excitonic effects. ${ }^{31}$ Also the importance of the LFE, in particular in transition-metal oxides with their localized $d$ states, could be shown by these calculations. ${ }^{18,23,24}$ It is therefore worthwhile to investigate more in detail to which extent calculations along these lines, that are rather efficient and moreover parameter-free, are able to give insight concerning IXS spectra of transition-metal oxides at large momentum transfer.

In this work we present IXS measurements of the dynamic structure factor in a range of large momentum transfer, and compare to theoretical results. In particular we show that already at the level of the random phase approximation (RPA) based on TDDFT a fair description of the observed $d$ - $d$ transitions in the IXS spectra of $\mathrm{V}_{2} \mathrm{O}_{3}$ can be obtained. LFE turn out to be the key ingredient to obtain a level of agreement between theory and experiment that is sufficient to interpret the results, understand the main spectral features of the metal-insulator transition, and predict features such as the effects of anisotropy. The surprising success of the RPA is due to a cancellation of many-body effects in the one- and two-particle Green's function that is not fortuitous but inherent in the theory.

After the presentation of experiments in Sec. II, we give a short introduction to the theoretical framework in Sec. III, where we also predict cancellation effects. Section IV is devoted to the presentation of the results, with a theoretical analysis of the measured spectra and a prediction for different directions of the momentum transfer. Finally, we conclude in Sec. V.

\section{EXPERIMENT}

The IXS measurements were carried out at 30-ID-XOR beamline, Advanced Photon Source, on the MERIX spectrometer working at $8.990 \mathrm{keV}^{32}$ To optimize the resolving power, the spectrometer was operated in the microdispersive geometry ${ }^{33}$ combining a spherically bent diced $\mathrm{Ge}(337)$ analyzer at near backscattering condition and a position-sensitive strip detector; the incident energy bandwidth was adequately reduced using a $\mathrm{Si}(004)$ high-resolution monochromator. The total resolution was estimated around $120 \mathrm{meV}$. The IXS spectra were recorded in a single crystal of $1.1 \%$-Cr-doped $\mathrm{V}_{2} \mathrm{O}_{3}$ in both paramagnetic metallic (PM) and paramagnetic insulating (PI). The sample was oriented with the $c$ axis along the scattering vector $\mathbf{Q}$ in the vertical plane and the $a$ axis parallel to the incident polarization. The PM-PI transition was induced by temperature change from 300 to $100 \mathrm{~K}$. The energy-loss spectra were obtained around the reciprocal 
lattice vector $(0,0,9)$, corresponding to a momentum transfer of $\mathbf{Q}=4.06 \AA^{-1}$. The IXS signal intensity was found to diminish significantly at lower $\mathbf{Q}$, while showing strong anisotropy: No IXS features were observed either with $\mathbf{Q}$ along the $a$ axis in vertical scattering or in the horizontal scattering geometry (Q $\mathbf{Q} \mathbf{a}$ or $\mathbf{c}$, in the horizontal plane).

\section{THEORY}

\section{A. Theoretical approaches to the description of the dynamic structure factor}

The dynamic structure factor contains the neutral excitations of the system. It is proportional to the imaginary part of the density-density response function $\chi$, since (1) can be written as

$$
S(\mathbf{Q}, \omega)=-\frac{\hbar^{2}}{\pi e^{2} n} \operatorname{Im} \chi(\mathbf{Q}, \omega) .
$$

Therefore it can be described alternatively as the linear response of the density to an external longitudinal perturbation, via time-dependent density functional theory (TDDFT), or as a particular contraction of the two-particle correlation function, which can be obtained from the Bethe-Salpeter equation (BSE). ${ }^{29}$

In the former case, one works entirely with Kohn-Sham quantities. The linear response function $\chi$ is defined as the variation of the density with respect to the external potential,

$$
\chi(1,2)=\frac{\delta n(1)}{\delta V_{\text {ext }}(2)}{ }_{\text {|equilibrium }},
$$

but TDDFT gives direct access to the dependence of the density on the total Kohn-Sham potential $V_{K S}$,

$$
\chi_{K S}(1,2)=\frac{\delta n(1)}{\delta V_{K S}(2)} \text { |equilibrium }_{\text {. }} .
$$

Here numbers represent space, spin, and time indices; e.g., $1=\left(\mathbf{r}_{1}, \sigma_{1}, t_{1}\right)$. The connection between the two quantities can be derived by inserting in Eq. (4) a functional derivative with respect to the density using the chain rule; this yields ${ }^{34,35}$

$$
\chi(1,2)=\chi_{K S}(1,2)+\chi_{K S}(1, \overline{3})\left[v_{c}(\overline{3}, \overline{4})+f_{x c}(\overline{3}, \overline{4})\right] \chi(\overline{4}, 2),
$$

where (repeated indices are integrated over)

$$
f_{x c}(3,4)=\frac{\delta V_{x c}(3)}{\delta n(4)}
$$

is the so-called exchange-correlation kernel and the contribution $v_{c}$, the Coulomb potential, stems from the variation of the Hartree potential. Fourier transform from time to frequency space leads to (space arguments are omitted for simplicity)

$$
\chi(\omega)=\chi_{K S}(\omega)+\chi_{K S}(\omega)\left[v_{c}+f_{x c}(\omega)\right] \chi(\omega) .
$$

The complexity of the interacting problem is hidden in the exchange-correlation potential and kernel.

Bethe-Salpeter calculations focus on the two-particle correlation function $L$, which is obtained from

$$
\begin{aligned}
L\left(1,2,1^{\prime}, 2^{\prime}\right)= & L_{0}\left(1,2,1^{\prime}, 2^{\prime}\right) \\
& +L_{0}\left(1, \overline{3}^{\prime}, 1^{\prime}, \overline{3}\right) \Xi\left(\overline{3}, \overline{2}, \overline{3}^{\prime}, \overline{2}^{\prime}\right) L\left(\overline{2}^{\prime}, 2, \overline{2}, 2^{\prime}\right),
\end{aligned}
$$

with

$$
\Xi\left(\overline{3}, \overline{2}, \overline{3}^{\prime}, \overline{2}^{\prime}\right)=-i \delta\left(\overline{3} \overline{3}^{\prime}\right) \delta\left(\overline{2}^{\prime}, \overline{2}\right) v_{c}(\overline{3} \overline{2})+\frac{\delta \Sigma_{x c}\left(\overline{3}, \overline{3}^{\prime}\right)}{\delta G\left(\overline{2}^{\prime}, \overline{2}\right)} .
$$

Here $G$ is the fully interacting one-body Green's function and

$$
L_{0}\left(1,2,1^{\prime}, 2^{\prime}\right)=G\left(1,2^{\prime}\right) G\left(2,1^{\prime}\right)
$$

is the result for the independent propagation of two dressed particles. The density-density response function is then obtained from $\chi(1,2)=L(1,2,1,2)$.

Note that the Kohn-Sham response $\chi_{K S}(1,2)$ can be expressed as

$$
\chi_{K S}(1,2)=L_{0}^{K S}(1,2,1,2) \equiv G_{K S}(1,2) G_{K S}(2,1),
$$

which is not the contraction of $L_{0}$ in Eq. (11), that is instead built with dressed Green's functions $G$. The kernel contribution $v_{c}$ in Eqs. (8) and (10) has the same origin in both approaches (namely, the density variation of the Hartree potential), and it plays the same role (i.e., its long-range contribution creates plasmons, and the microscopic parts are responsible for crystal local field effects). The derivative of the self-energy $\Sigma_{x c}$ in the Bethe-Salpeter equation contains the electron-hole attraction. In the case of TDDFT, the exchange-correlation kernel $f_{x c}$ implicitly simulates the global effect of the difference between $L_{0}$ and $L_{0}^{K S}$ and the derivative of $\Sigma_{x c}$.

\section{B. RPA or beyond?}

When $f_{x c}$ is neglected in the TDDFT linear response Dyson equation (8) one obtains the random phase approximation (RPA). ${ }^{43}$ One may suspect this to be a very rough approximation; however, it may be justified in cases where the self-energy corrections to the Kohn-Sham band structure and the effects of the electron-hole attraction cancel each other to a large extent. Indeed, whereas it is obvious that both contributions can be neglected when they are small, one should not forget that they can also be neglected when they cancel each other, even when they are very strong. This cancellation has not been explored very much, and it is one of the main points of the present work. Let us therefore recall some facts.

First, take the case of the excitation of one single electron. There are of course no exchange or correlation effects in the optical spectrum of such a system. However, as has been discussed in detail in Ref. 29, a Bethe-Salpeter calculation of the problem would require a true many-body calculation, because via $L_{0}$, and hence $G$, it contains the addition of a second electron as an intermediate step. Thanks to the Coulomb repulsion, in a potential that localizes the electrons significantly one would find a quite large quasiparticle (i.e., electron addition and removal) gap when Hartree and selfenergy effects are included, and a much smaller optical gap, that corresponds to the independent-particle one, due to the "excitonic effects" that in this example perfectly cancel the former contributions. A TDDFT calculation of the same problem also shows cancellations (between Hartree and exchange potential, and between $v_{c}$ and $f_{x c}$ in the kernel), but the contributions are smaller because there is no electron addition. Indeed, in this case $\chi_{K S}$ is already equal to the exact solution. Besides these quantitative considerations, one should also keep in mind the qualitative complications that one may 
encounter when choosing the Bethe-Salpeter way. Take the two-site Hubbard model with one electron, simulating the $\mathrm{H}_{2}^{+}$ molecule. This system is "strongly correlated": In particular, in the atomic limit electron addition will lead to a double peak in the spectral function (the additional electron may, or may not, meet the already present one, which leads to an energy difference given by the repulsion $U$ ) whereas any standard mean field approach such as Hartree-Fock or even certain many-body perturbation approaches such as the popular GW approximation will find only one peak, determined by the average $U / 2 .{ }^{36}$ Hence, a good Bethe-Salpeter calculation would require a difficult description of correlation effects, well beyond standard approximations, whereas the exact result for the optical excitations can be obtained by a simple noninteracting calculation.

Also note that the interpretation of the results is inherently different when the full Bethe-Salpeter or an RPA or timedependent local density approximation (TDLDA) approach are used, even though spectra might be very similar. One can understand this looking at a static approximation to the BSE. Then the result can be obtained by diagonalizing a two-particle Hamiltonian in a basis of transitions between quasiparticle states. These transitions are mixed due to the electron-hole attraction. Again, one can see from the one-electron limit that indeed a mixing would be found, whereas the exact result is a sum of independent transitions. The clue is that the latter transitions occur between noninteracting states, so that there is no contradiction.

These considerations illustrate that the calculation of neutral excitations of so-called correlated systems should not necessarily be performed in the same framework as the calculation of, e.g., photoemission, and that advanced methods yielding a good one-particle Green's function might fail, or at least be worse than a lower level calculation, for the calculation of two-particle excitations, unless a consistent kernel for the linear response calculation is available that correctly leads to the cancellations. In any case, such calculations can be suspected to be much more involved than what one would actually need, and it is certainly worthwhile to investigate alternatives, starting from the simple random phase approximation.

Real systems contain more than one electron and one cannot hope to get away with a completely noninteracting framework. However, in finite systems such as small molecules or clusters simple approximations to TDDFT turn out to work surprisingly well, with huge cancellations when compared to a Bethe-Salpeter calculation. This has been observed, e.g., for a small sodium cluster ${ }^{37,38}$ or silicon nanocrystals. ${ }^{39}$ Here the adiabatic local density approximation (TDLDA) does a very good job. Moreover, the effect of $f_{x c}$ in the TDLDA is weak, so that even an RPA calculation leads to acceptable results, as in many other comparable systems. Cancellations between self-energy and excitonic effects have also been observed for carbon nanotubes ${ }^{40,41}$ as well as on the plasmon peak position in solids. ${ }^{42}$

Here we are interested in localized excitations that are contained in the dynamic structure factor. The electrons that contribute mainly to these excitations are similar to particles in a box, or in finite systems. Indeed, as we will see later the loss function (that typically displays plasmons) and the imaginary part of the macroscopic dielectric function (that features interband transitions and excitons) are very similar in the energy range of interest, just as in a finite system. ${ }^{29}$ One can hence expect large self-energy and electron-hole corrections, with significant cancellations.

This hypothesis is further supported by setting up a simple model self-energy representing the main characteristics of the electronic structure of $\mathrm{V}_{2} \mathrm{O}_{3}$ that we need here. Concerning the metallic phase, to first approximation one can remain on an LDA level because the many-body effects are strongly screened, and because we are not interested in satellites here. The problem is the insulating phase, where LDA wrongly yields a metal. DMFT calculations ${ }^{12}$ show that in the band structure the biggest difference between the paramagnetic metal and the paramagnetic insulator is a rigid downshift of the occupied flat $t_{2 g}$ bands close to the Fermi level, which leads to the gap opening. This downshift is completely absent in LDA calculations, whereas other features further away from the Fermi level are correctly reproduced, in particular the broadening of the unoccupied $e_{g}^{\sigma}$ bands, as we will see later in more detail. This suggests the following static model self-energy:

$$
\Sigma_{x c}=v_{x c}-\Delta \sum_{v}|v\rangle\langle v|,
$$

where $\Delta$ is a positive constant and $|v\rangle$ are occupied states. We suppose that the densities stemming from the Kohn-Sham potential $v_{x c}$ and from this self-energy are very similar so that we can neglect the difference, which is the case in particular when the quasiparticle and Kohn-Sham occupied states are very similar. This model is restricted to the localized $d$ states; as in DMFT calculations, for now we suppose that these can be decoupled from the other states that then merely yield a background screening.

The above model for $\Sigma_{x c}$ can now be expressed in terms of the dressed Green's function $G$ :

$$
\begin{aligned}
& \Sigma_{x c}\left(\mathbf{r}, \mathbf{r}^{\prime}, t, t^{\prime}\right) \\
& \quad=\delta\left(t^{\prime}-t^{+}\right)\left[\delta\left(\mathbf{r}-\mathbf{r}^{\prime}\right) v_{x c}(\mathbf{r})+i \Delta G\left(\mathbf{r}, \mathbf{r}^{\prime}, t, t^{\prime}\right)\right] .
\end{aligned}
$$

The exchange-correlation contribution to the kernel of the Bethe-Salpeter equation reads then ${ }^{44}$

$$
\begin{aligned}
& \Xi_{x c}\left(\mathbf{r}_{1}, \mathbf{r}_{2}, t_{1}, t_{2} ; \mathbf{r}_{3}, \mathbf{r}_{4}, t_{3}, t_{4}\right) \\
& =\frac{\delta \Sigma_{x c}\left(\mathbf{r}_{1}, \mathbf{r}_{2}, t_{1}, t_{2}\right)}{\delta G\left(\mathbf{r}_{3}, \mathbf{r}_{4}, t_{3}, t_{4}\right)} \\
& =\delta\left(t_{2}-t_{1}^{+}\right)\left[-i \delta\left(\mathbf{r}_{1}-\mathbf{r}_{2}\right) \delta\left(\mathbf{r}_{3}-\mathbf{r}_{4}\right) \frac{\delta v_{x c}\left(\mathbf{r}_{1}, t_{1}\right)}{\delta \rho\left(\mathbf{r}_{3}, t_{3}\right)} \delta\left(t_{3}-t_{4}\right)\right. \\
& \left.\quad+i \Delta \delta\left(\mathbf{r}_{1}-\mathbf{r}_{3}\right) \delta\left(\mathbf{r}_{2}-\mathbf{r}_{4}\right) \delta\left(t_{1}-t_{3}\right) \delta\left(t_{2}-t_{4}\right)\right]
\end{aligned}
$$

which leads to matrix elements of the electron-hole interaction in transition space $(|c\rangle$ are empty states)

$$
\begin{aligned}
\Xi_{x c(v c)}^{\left(v^{\prime} c^{\prime}\right)}\left(t_{1}, t_{2} ; t_{3}, t_{4}\right)= & \delta\left(t_{2}-t_{1}^{+}\right) \delta\left(t_{3}-t_{4}\right)\left[f_{x c(v c)}^{\left(v^{\prime} c^{\prime}\right)}\left(t_{1}, t_{3}\right)\right. \\
& \left.-\Delta \delta_{\left(v v^{\prime}\right)} \delta_{\left(c c^{\prime}\right)} \delta\left(t_{1}-t_{3}\right)\right] .
\end{aligned}
$$

In other words, we have the usual (eventually dynamical) TDDFT contribution of the local KS potential, ${ }^{45}$ plus a static contribution that is diagonal in the transition space, and that hence simply decreases excitation energies by the value of 
$\Delta:$ The electron-hole contribution of the nonlocal part of the self-energy completely cancels the self-energy shift. This is what should happen in the case of the exact $v_{x c}$ and $\Sigma_{x c}$, since then already the TDDFT two-particle equation, constructed using KS eigenvalues, must give the exact result. However, it also shows that an approximate TDDFT calculation will yield good result in cases where the self-energy correction is essentially a rigid gap opening $\Delta$ without significant change in the density, even when the absolute value of $\Delta$ is very big. For the case in which this approximation is the TDLDA, $f_{x c}$ has usually little effect and can also be neglected, which brings us back to the RPA. The latter still contains the Coulomb contribution $v_{c}$ in the linear response Dyson equation (6) that should a priori be taken into account; indeed, as we will see in the following it plays a very important role in the dynamic structure factor of $\mathrm{V}_{2} \mathrm{O}_{3}$.

Before coming to the results, it should be stressed that the above-discussed cancellations are not to be considered as error canceling. There is by definition nothing such as self-energy corrections and electron-hole interaction in TDDFT, since no additional charges are present in this theoretical approach, just as they are not present in experiment. In a way, the BSE approach can be considered as a longer way, containing a detour, that usually makes the design of approximations easier but calculations more complicated. In the particular case of the $d$ - $d$ excitations considered here, it may well turn out that simple approximations of TDDFT outperform approximations of the BSE, because the detour is particularly complicated for correlated materials. Instead, when mixed approaches are used such as LDA $+U$ for the band structure calculations (which can be assimilated to an approximate calculation of $L_{0}$, rather than $L_{0}^{K S}$ ), but no electron-hole interaction kernel is taken into account, the results are, not surprisingly, satisfactory in energy and momentum transfer ranges where excitonic effects are weak, but disappointing because of an inconsistency when they are strong. ${ }^{19,20}$

\section{RESULTS}

\section{A. Signature of the PM-PI transition}

The change of the electronic structure across the MIT in $\mathrm{V}_{2} \mathrm{O}_{3}$ occurs prominently for the $d$ states around the Fermi level. Thus the MIT can be studied by searching for the spectral structures associated with the $d-d$ transitions and analyzing their variation across the MIT. Experimental IXS spectra [see Fig. 1(a)] show a prominent tail at small energies that is due to the elastic scattering of photons. There are different possibilities to remove this elastic contribution from the spectra, which would introduce a large error bar. We have hence reported here the raw experimental data, where, besides the elastic tail, a peak is found in both phases at $2-4 \mathrm{eV}$ for a momentum transfer $\mathbf{Q} \sim 4.06 \AA^{-1}$ in the $c$ direction of the hexagonal unit cell. We focus on this peak: Its modification bears the signature of the MIT. In the PI phase [blue diamonds in Fig. 1(a)] the peak is located between 1.9 and $3.7 \mathrm{eV}$, while for the PM phase [red circles in Fig. 1(a)] the same structure is blueshifted by $\sim 0.3 \mathrm{eV}$ and has a lower intensity. ${ }^{46}$ Our calculations, ${ }^{49}$ shown in Fig. 1(b), reproduce this peak, though its absolute position is slightly overestimated. Most
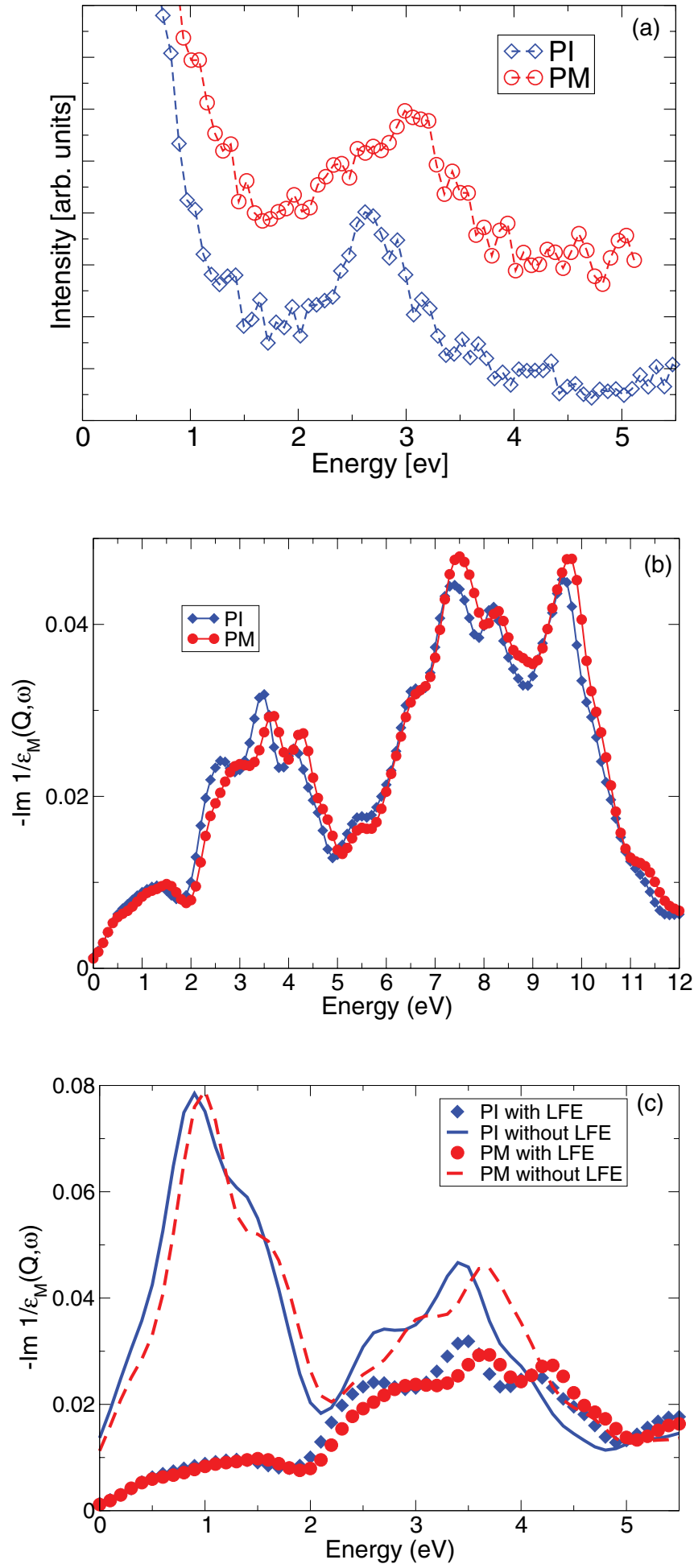

FIG. 1. (Color online) IXS spectra of the dynamic structure factor $S(\mathbf{Q}, \omega) \propto-\operatorname{Im} 1 / \epsilon_{M}(\mathbf{Q}, \omega)$ at $\mathbf{Q}=4.06 \AA^{-1}$ for the PI (blue diamonds) and the PM (red circles) phases, at $\mathbf{Q}=4.06 \AA^{-1}$. (a) Experiment. The elastic peak has not been removed from the spectra. (b) Results of an RPA calculation (note the extended energy range). (c) Comparison of RPA spectra in the low-energy range with (PI, blue diamonds, and PM, red circles) and without (PI, blue solid line and PM, red dashed line) crystal local field effects. The main effect of the crystal local fields is to suppress the first peak, which is indeed absent in the experiment. 
importantly, both the blueshift and the damping when going to the metallic phase are correctly reproduced. We also find the prominent structure below $10 \mathrm{eV}$ that was already measured in Ref. 21 for the antiferromagnetic phase. ${ }^{47}$ In view of this agreement, we can now use our calculations in order to analyze the spectra and link them to the band structure of $\mathrm{V}_{2} \mathrm{O}_{3}$.

From the theoretical point of view, the modification of the electronic structure across the MIT can be seen already at the level of the joint density of states:

$$
\operatorname{JDOS}(\mathbf{q}, \omega)=\sum_{v c \mathbf{k}} \delta\left(\omega-\left(\varepsilon_{c \mathbf{k}}-\varepsilon_{v \mathbf{k}-\mathbf{q}}\right)\right),
$$

where $\mathbf{q}$ belongs to the first Brillouin zone, and $\mathbf{Q}=\mathbf{q}+\mathbf{G}$ along the $c$ axis with $\mathbf{G}$ a reciprocal lattice vector. In the DOS calculated with LDA band structure energies $\varepsilon_{n \mathbf{k}}$ [see Fig. 2(a)] for both phases we find two main structures, which are due respectively to $t_{2 g}$ states around the Fermi energy and to $e_{g}^{\sigma}$ states at higher energy. This leads to the JDOS with two peaks centered at $\sim 1 \mathrm{eV}$ and $\sim 3.25-3.5 \mathrm{eV}$, respectively [see Fig. 2(b)]. From our analysis we have that $t_{2 g} \rightarrow t_{2 g}$ transitions around the Fermi energy give rise to the first peak at $1 \mathrm{eV}$. The second peak instead stems from $t_{2 g} \rightarrow e_{g}^{\sigma}$ transitions. In the metal this peak is shifted to higher energies and has a lower intensity, as in the experiment. The origin of the difference of the JDOS between the two phases can be understood from
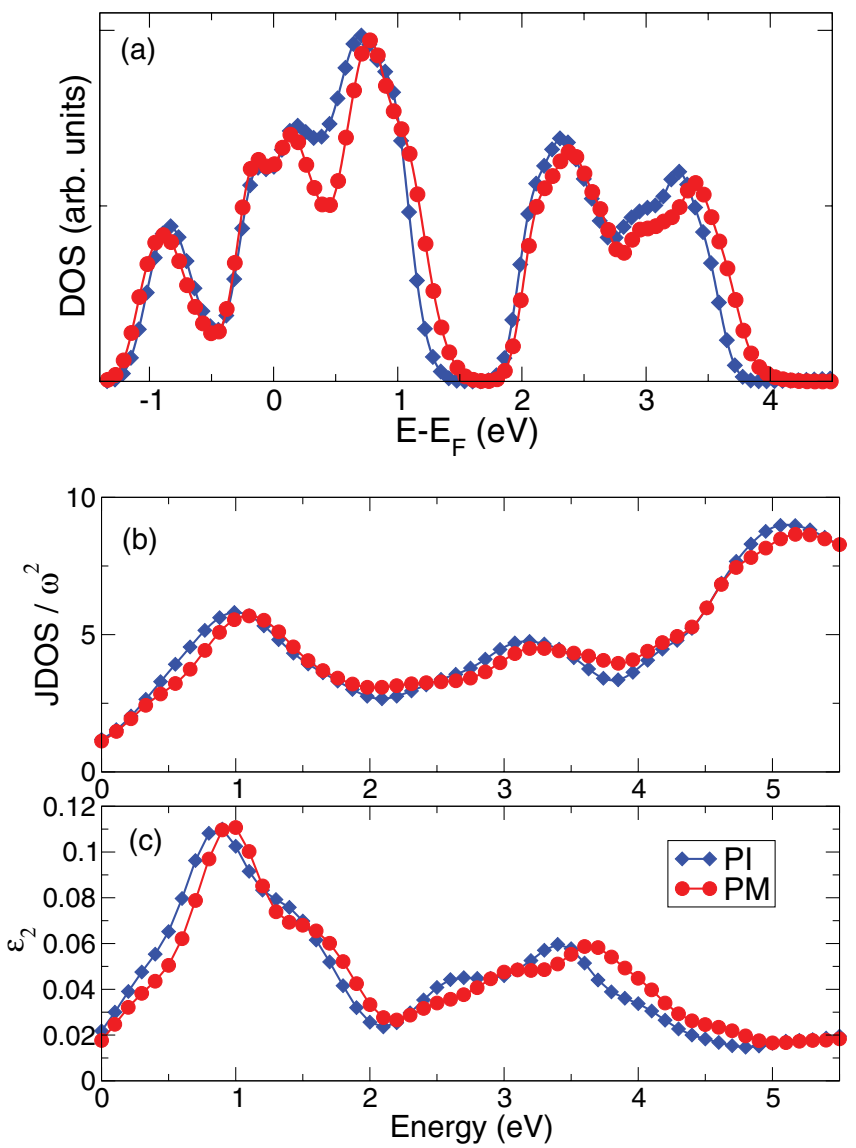

FIG. 2. (Color online) (a) LDA density of states, (b) JDOS $/ \omega^{2}$, and (c) imaginary part of the macroscopic dielectric function $\varepsilon_{2}$ calculated in the RPA without LFE at $\mathbf{Q}=4.06 \AA^{-1}$ for the PI (blue diamonds) and PM (red circles) phases. the modification of their band structure energies (see, e.g., Ref. 10). The $t_{2 g}$ and $e_{g}^{\sigma}$ bandwidths are larger in the PM phase, where the intensity of the peaks in the DOS is correspondingly reduced. Moreover, the unoccupied $e_{g}^{\sigma}$ bands are slightly shifted towards the Fermi energy in the PI phase. Thus, the comparison of the LDA band structures already explains the difference in the second JDOS peak and the variation of the experimental results across the MIT.

However, the JDOS spectra do not compare well with the experiment. The first peak at $\sim 1 \mathrm{eV}$ is not present in the experimental IXS spectra. Moreover, the second peak, which corresponds to the structure measured in the experiment, appears actually to be only a shoulder of a more intense peak after $4 \mathrm{eV}$ where the JDOS is strongly rising. Therefore, we have to go beyond the description of the spectra based on the JDOS, in order to get a better agreement with the experiment and verify that the interpretation of spectral signatures of the MIT remains valid.

In addition to the band structure energies, in the calculation of the spectra a second ingredient is the consideration of the oscillator strength matrix elements: $\rho_{v, c}^{\mathbf{k}}(\mathbf{Q})=\langle v, \mathbf{k}-$ $\left.\mathbf{q}\left|e^{-i \mathbf{Q} \cdot \mathbf{r}}\right| c, \mathbf{k}\right\rangle$. The combination of the JDOS with the matrix elements gives the imaginary part of the dielectric function $\epsilon_{2}(\mathbf{Q}, \omega)$, at the level of the RPA without LFE, $\epsilon_{2}=-v_{c} \operatorname{Im} \chi_{K S}$. The main effect of the matrix elements in $\epsilon_{2}$ is to cancel the rise of the JDOS after the second peak [see Fig. 2(c)], in agreement with the experiment. The other features of the spectra are similar to the JDOS results, with a small redshift of the first peak in $\epsilon_{2}$ and a small increase of the difference between the two phases for the second peak. Indeed, the spectrum would be suppressed at small $\mathbf{Q}$ where dipole selection rules apply, but this is not the case for the large $\mathbf{Q}$ considered here.

The spectra of $\epsilon_{2}(\mathbf{Q}, \omega)$ and the calculated dynamic structure factor $S(\mathbf{Q}, \omega)$ are very similar. In fact, at the large momentum transfer that we are considering here, the real part of the dielectric function $\epsilon_{1}(\mathbf{Q}, \omega)$ is flat and numerically close to 1. So $S(\mathbf{Q}, \omega) \propto-\operatorname{Im} 1 / \epsilon_{M}(\mathbf{Q}, \omega) \simeq \epsilon_{2}(\mathbf{Q}, \omega)$. Therefore also $S(\mathbf{Q}, \omega)$ calculated without LFE has two peaks [see blue solid and red dashed lines in Fig. 1(c) for PI and PM, respectively] instead of one as in the experiment. Instead, when LFE are taken into account, the first spurious peak at $\sim 1 \mathrm{eV}$ is strongly damped and almost suppressed [see diamonds and circles in Fig. 1(c)], leading to a much better agreement with the experimental spectra for both phases. Indeed, the second peak is much less affected by the LFE, and the signature of the MIT stemming from the JDOS remains clearly visible in the spectra. Therefore, LFE are crucial to obtain a safe comparison with the experiment. This comparison confirms the interpretation of the fingerprint of the transition based on the JDOS.

LFE are linked to the microscopic part of the Coulomb potential $v_{c}$, i.e., to induced Hartree interactions due to microscopic charge inhomogeneities. Therefore they play a fundamental role in inhomogeneous and polarizable materials, especially when the short-range spatial scale is probed in the experiment. This is precisely what occurs in the present case in which the measurement at a large momentum transfer corresponds to a local probe in real space. This is the spatial scale where the localized $d$ states give rise to inhomogeneous charge densities that can be polarized quite effectively. LFE 
are responsible for the strong mixing of independent $d$-d transitions, with a smaller contribution also from transitions involving $\mathrm{O} p$ states at higher energies. The result is a spectrum in which each structure is a combination of several contributions and cannot be assigned to single independentparticle transitions.

With respect to experiment, the calculated peaks are still too broad and their energy is slightly overestimated. The shrinking of these peaks derives from a correction to the LDA DOS, visible, e.g., in the $t_{2 g}$ bandwidth reduction obtained in DMFT. ${ }^{12}$ It is similar for the two phases, and would hence not change our conclusions. The overall good agreement with the experiment demonstrates that the RPA is already sufficient to explain the main spectral features across the MIT, once the LFE have been taken into account, although the differences between the two phases in the position and shape of the peaks are underestimated in the calculation. This result confirms the expectations deriving from the model static self-energy (13) for a cancellation between different correlation effects on the one- and two-particle Green's functions, as has been discussed in Sec. III B.

The same strong cancellation effects have been found also in the IXS spectra at large momentum transfer in $\mathrm{NiO}^{19,20}$ supporting our conclusions for the PI phase of $\mathrm{V}_{2} \mathrm{O}_{3}$. Also in that case, RPA calculations using an LDA $+U$ band structure are in agreement with the experiment concerning the presence of a sharp peak due to $d$ - $d$ excitations. ${ }^{19}$ However, the position of the peak in the calculated spectra is shifted by $\sim 5 \mathrm{eV}$ to higher energy with respect to the experiment. This difference corresponds to the binding energy due to the electron-hole attraction, not included in the calculation. It is of the same order of magnitude as the value of the Hubbard $U$ ( $U=$ $8 \mathrm{eV}$ ) adopted in the underlying LDA $+U$ band structure calculation, which was used to build the RPA response function and obtain the spectra in NiO. The main effect of the Hubbard $U$ with respect to the LDA DOS in $\mathrm{NiO}$ is in fact a rigid opening (with increasing $U$ ) of the band gap between the $d$ states that give rise to the peak in the IXS. ${ }^{53}$ Indeed, the $d$ - $d$ transitions across LDA band gap (which is $\sim 0.7 \mathrm{eV}$ ) are located much closer to the peak measured in IXS (which starts at $\sim 1 \mathrm{eV}$ ) than those across the LDA $+U$ band gap.

\section{B. Spatial anisotropy}

With these considerations in mind we can now investigate more details of the spectra. Here we are in particular interested in the anisotropic character of $\mathrm{V}_{2} \mathrm{O}_{3}$. Microscopic charge inhomogeneities in the materials can strongly vary in different spatial directions, leading to anisotropic spectra. ${ }^{54}$ Moreover, the anisotropy of the polarizability of these inhomogeneities influences the LFE. Therefore spatial anisotropies in the density response can be put into evidence by the different behavior of the LFE for spectra calculated for momentum transfers along different directions. In fact, LFE are enhanced by strong spatial variations of the charge densities with respect to their spatial average. ${ }^{18}$ On the basis of our calculations, we predict that $\mathrm{V}_{2} \mathrm{O}_{3}$ shows a strong anisotropy, as we now discuss for the PI phase (results for the PM phase are similar).

In addition to the momentum transfer $\mathbf{Q}=\mathbf{Q}_{c}$ along the hexagonal $c$ axis, we have calculated the dynamic structure

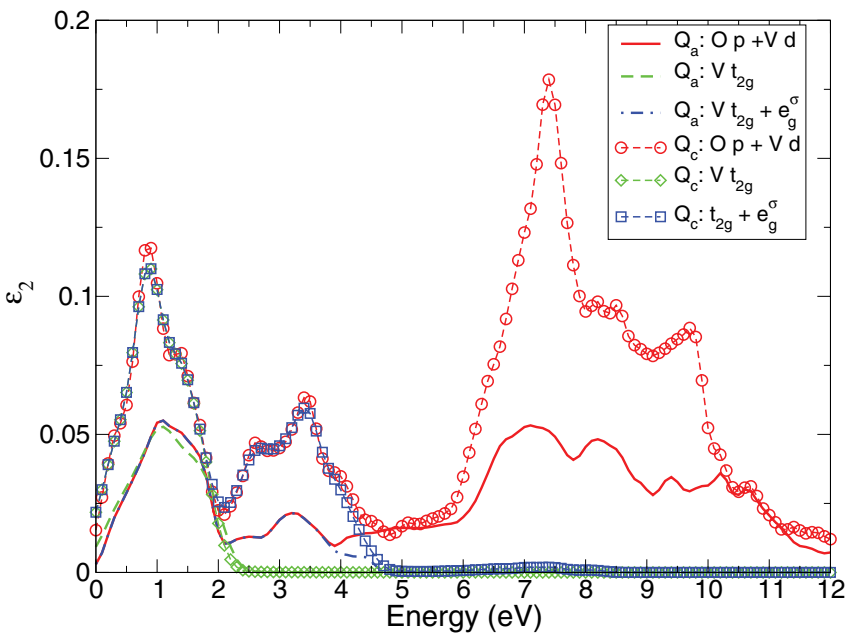

FIG. 3. (Color online) Imaginary part of the macroscopic dielectric function $\epsilon_{2}(\mathbf{Q}, \omega)=\operatorname{Im} \epsilon_{M}(\mathbf{Q}, \omega)$, calculated in the PI phase for two momentum transfers $\mathbf{Q}_{c}=(0,0,9)\left(4.06 \AA^{-1}\right)$ and $\mathbf{Q}_{a}=$ $(3,0,0)\left(4.35 \AA^{-1}\right)$ (symbols and lines, respectively) in the hexagonal Brillouin zone, without local-field effects (LFE). Green diamonds and dashed line: Only $\mathrm{V} t_{2 g}$ states are taken into account. Blue squares and dot-dashed: In addition, $e_{g}^{\sigma}$ are included. Red circles and continuous: Finally, O $2 p$ states are added.

factor $S(\mathbf{Q}, \omega)$ also along two other directions in the $a b$ plane perpendicular to the $c$ axis: $\mathbf{Q}_{a}=(3,0,0)$ and $\mathbf{Q}_{a b}=$ $(5 / 3,5 / 3,0)$ in the Brillouin zone of the hexagonal cell.

Let us first analyze which states contribute to the various spectral features. To this end, we compare in Fig. 3 calculations for the $c$ and $a$ directions, for the sake of simplicity for $\epsilon_{2}$ without LFE. Then the spectrum is a simple sum over transitions. Adding or excluding groups of transitions will then tell us which parts of the spectra are influenced by the corresponding states. Results in the $c$ direction are given by symbols, results in the $a$ direction by lines. When only $\mathrm{V} t_{2 g}$ states are taken into account (diamonds and dashed line), the spectra show the first peak at low energy that, as we have seen earlier for the $c$ direction, is then partially suppressed by LFE. When $e_{g}^{\sigma}$ states are included (squares and dot-dashed), the peak around $3 \mathrm{eV}$, which is our main interest, appears. Features at higher energies are due to the $\mathrm{O} 2 p$ states (circles and continuous line). There is a big difference between the two directions at all energies: Moving from $c$ to $a$, the intensity is decreased by more than a factor of two at all energies. The peak around $3 \mathrm{eV}$ is particularly affected, but altogether the spectral shape remains similar.

In Fig. 4 we compare results with and without LFE; now the $a b$ direction is added (whereas results for $c$ can be found in Fig. 1). Here results in the $a b$ direction are given by symbols, results in the $a$ direction by lines. In the $a b$ direction the system behaves very similarly to the $c$ direction, with a strong suppression of the first peak and a more moderate decrease on the second peak. Instead, in the $a$ direction the first peak is much less affected. The fact that LFE are much less relevant in that case indicates that in the $a$ direction the electronic charge is more homogeneous than for $\mathbf{Q}_{a b}$. Also LFE on the second, weak peak are minor in the $a$ direction. Since the intensity for $\mathbf{Q} \| a$ without LFE is already very small compared to the other 


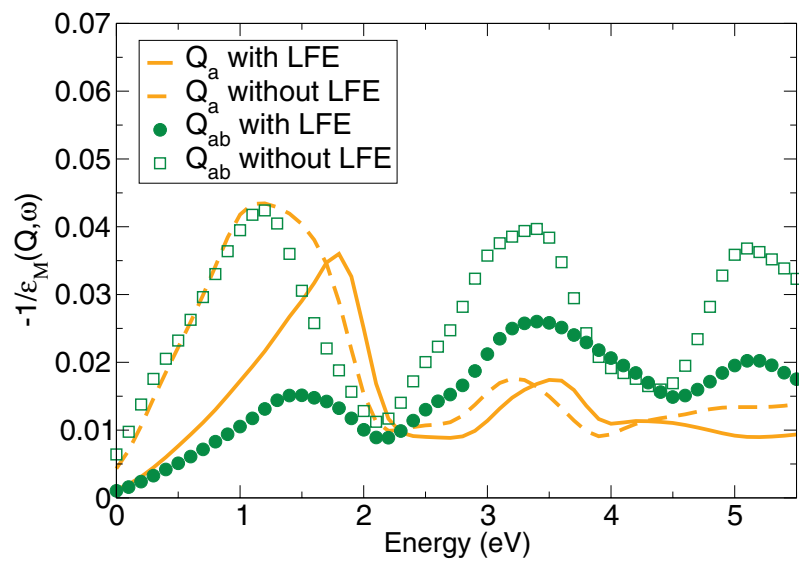

FIG. 4. (Color online) Dynamic structure factor $S(\mathbf{Q}, \omega) \propto$ $-\operatorname{Im} 1 / \epsilon_{M}(\mathbf{Q}, \omega)$, calculated in the PI phase for two in-plane momentum transfers $\mathbf{Q}_{a b}=(5 / 3,5 / 3,0)\left(4.23 \AA^{-1}\right)$ and $\mathbf{Q}_{a}=(3,0,0)$ $\left(4.35 \AA^{-1}\right)$ in the hexagonal Brillouin zone. Results in the $a b$ direction are given by symbols (circles and squares with and without LFE, respectively), results in the $a$ direction by lines (continuous and dashed lines with and without LFE, respectively). The spectral shape is highly anisotropic once LFE are included in the calculation.

directions, this might result from a reduced polarizability of the corresponding charge density. In any case, no significant structure remains around $3 \mathrm{eV}$ for $\mathbf{Q} \| a$, in agreement with experiment. To be precise, the measured spectra (not shown) do not exhibit any intensity in that range and direction, whereas a small bump remains in the calculation. This might be due to errors induced by the LDA; it is in fact known that LDA has a tendency to underestimate anisotropies. ${ }^{59}$ Overall, our results show that the understanding of the anisotropy in the density response of $\mathrm{V}_{2} \mathrm{O}_{3}$ requires the inclusion of the LFE. ${ }^{23,56-58}$ Their importance is hence not only quantitative, but even qualitative.

\section{CONCLUSIONS}

In conclusion, we have presented experimental and theoretical results for the $d$ - $d$ transitions in the dynamic structure factor of $\mathrm{V}_{2} \mathrm{O}_{3}$. We have discussed different theoretical approaches, and argued that due to cancellations of many-body effects the simple random phase approximation, on a density-functional basis, constitutes an approach that allows one technically to perform parameter-free calculations for the correlated oxides, and that yields results for IXS at large momentum transfer that are reliable enough to understand measured data and predict trends. This is contrast to photoemission spectroscopy, where dynamical correlation plays a prominent role in the transfer of spectral weight across the metal-insulator transition. This finding has a general validity, suggesting that instead of classifying a material as "correlated" in general, one should rather discuss specific correlation effects that may manifest differently in the various electronic spectra of the material.

Moreover, here we have shown that crystal local field effects are an essential ingredient in order to obtain calculated spectra that are comparable to experimental ones. The experimental results for $\mathrm{V}_{2} \mathrm{O}_{3}$ evidence the fingerprint of the paramagnetic metal-insulator transition in the low-energy range, and our calculations elucidate the origin of the observations. A strong anisotropy is predicted by the calculations, and it is shown that this effect can only be found when crystal local field effects are taken into account.

\section{ACKNOWLEDGMENTS}

Use of the Advanced Photon Source at Argonne National Laboratory was supported by the US Department of Energy, Office of Science, Office of Basic Energy Sciences, under Contract No. DE-AC02-06CH11357. We are grateful for support by ETSF-I3 Grant No. 211956. Computer time was granted by IDRIS (544). F.I. also acknowledges financial support from the CEA program Transversal Nanosciences and M.G. from the European Research Council Advanced Grant DYNamo (ERC-2010-AdG Proposal No. 267374), Spanish Grants No. FIS2011-65702-C02-01 and No. PIB2010US00652, ACI-Promociona (ACI2009-1036), Grupos Consolidados UPV/EHU del Gobierno Vasco (IT-319-07), Consolider nanoTHERM (Grant No. CSD2010-00044), and European Commission projects CRONOS (280879-2 CRONOS CPFP7) and THEMA (FP7-NMP-2008-SMALL-2, 228539).

\footnotetext{
*Present address: Dipartimento di Scienze e Metodi dell'Ingegneria, Università di Modena e Reggio Emilia, Via Amendola 2 Padiglione Morselli, I-42122 Reggio Emilia, Italy.

†Present address: Material Science Division, Argonne National Laboratory, Argonne, Illinois 60439, USA.

${ }^{1}$ N. Mott, Metal-Insulator Transitions, 2nd ed. (Taylor \& Francis, London, 1990).

${ }^{2}$ M. Imada, A. Fujimori, and Y. Tokura, Rev. Mod. Phys. 70, 1039 (1998).

${ }^{3}$ D. B. McWhan, T. M. Rice, and J. P. Remeika, Phys. Rev. Lett. 23, 1384 (1969); D. B. McWahn, A. Menth, J. P. Remeika, W. F. Brinkman, and T. M. Rice, Phys. Rev. B 7, 1920 (1973).

${ }^{4}$ M. J. Rozenberg, G. Kotliar, H. Kajueter, G. A. Thomas, D. H. Rapkine, J. M. Honig, and P. Metcalf, Phys. Rev. Lett. 75, 105 (1995).

${ }^{5}$ J.-H. Park, L. H. Tjeng, A. Tanaka, J. W. Allen, C. T. Chen, P. Metcalf, J. M. Honig, F. M. F. de Groot, and G. A. Sawatzky, Phys. Rev. B 61, 11506 (2000)

${ }^{6}$ F. Rodolakis, P. Hansmann, J.-P. Rueff, A. Toschi, M. W. Haverkort, G. Sangiovanni, A. Tanaka, T. Saha-Dasgupta, O. K. Andersen, K. Held, M. Sikora, I. Alliot, J.-P. Itié, F. Baudelet, P. Wzietek, P. Metcalf, and M. Marsi, Phys. Rev. Lett. 104, 047401 (2010).

${ }^{7}$ S. Lupi, L. Baldassarre, B. Mansart, A. Perucchi, A. Barinov, P. Dudin, E. Papalazarou, F. Rodolakis, J.-P. Rueff, J.-P. Itié, S. Ravy, D. Nicoletti, P. Postorino, P. Hansmann, N. Parragh, A. Toschi, T. Saha-Dasgupta, O. K. Andersen, G. Sangiovanni, K. Held, and M. Marsi, Nature Commun. 1, 105 (2010).

${ }^{8}$ F. Rodolakis, J. P. Rueff, M. Sikora, I. Alliot, J. P. Itié, F. Baudelet, S. Ravy, P. Wzietek, P. Hansmann, A. Toschi, M. W. Haverkort, G. Sangiovanni, K. Held, P. Metcalf, and M. Marsi, Phys. Rev. B 84, 245113 (2011).
} 
${ }^{9}$ S. K. Mo, J. D. Denlinger, H. D. Kim, J. H. Park, J. W. Allen, A. Sekiyama, A. Yamasaki, K. Kadono, S. Suga, Y. Saitoh, T. Muro, P. Metcalf, G. Keller, K. Held, V. Eyert, V. I. Anisimov, and D. Vollhardt, Phys. Rev. Lett. 90, 186403 (2003).

${ }^{10}$ G. Keller, K. Held, V. Eyert, D. Vollhardt, and V. I. Anisimov, Phys. Rev. B 70, 205116 (2004).

${ }^{11}$ G. Panaccione, M. Altarelli, A. Fondacaro, A. Georges, S. Huotari, P. Lacovig, A. Lichtenstein, P. Metcalf, G. Monaco, F. Offi, L. Paolasini, A. Poteryaev, M. Sacchi, and O. Tjernberg, Phys. Rev. Lett. 97, 116401 (2006).

${ }^{12}$ A. I. Poteryaev, J. M. Tomczak, S. Biermann, A. Georges, A. I. Lichtenstein, A. N. Rubtsov, T. Saha-Dasgupta, and O. K. Andersen, Phys. Rev. B 76, 085127 (2007).

${ }^{13}$ E. Papalazarou, M. Gatti, M. Marsi, V. Brouet, F. Iori, L. Reining, E. Annese, I. Vobornik, F. Offi, A. Fondacaro, S. Huotari, P. Lacovig, O. Tjernberg, N. B. Brookes, M. Sacchi, P. Metcalf, and G. Panaccione, Phys. Rev. B 80, 155115 (2009).

${ }^{14}$ F. Rodolakis, B. Mansart, E. Papalazarou, S. Gorovikov, P. Vilmercati, L. Petaccia, A. Goldoni, J. P. Rueff, S. Lupi, P. Metcalf, and M. Marsi, Phys. Rev. Lett. 102, 066805 (2009).

${ }^{15}$ G. Panaccione, M. Sacchi, P. Torelli, F. Offi, G. Cautero, R. Sergo, A. Fondacaro, C. Henriquet, S. Huotari, G. Monaco, and L. Paolasini, J. Electron Spectrosc. Relat. Phenom. 156, 64 (2007).

${ }^{16}$ S. L. Adler, Phys. Rev. 126, 413 (1962); N. Wiser, ibid. 129, 62 (1963).

${ }^{17}$ I. G. Gurtubay, J. M. Pitarke, W. Ku, A. G. Eguiluz, B. C. Larson, J. Tischler, P. Zschack, and K. D. Finkelstein, Phys. Rev. B 72, 125117 (2005).

${ }^{18}$ F. Aryasetiawan, O. Gunnarsson, M. Knupfer, and J. Fink, Phys. Rev. B 50, 7311 (1994).

${ }^{19}$ B. C. Larson, W. Ku, J. Z. Tischler, C.-C. Lee, O. D. Restrepo, A. G. Eguiluz, P. Zschack, and K. D. Finkelstein, Phys. Rev. Lett. 99, 026401 (2007).

${ }^{20}$ C.-C. Lee, H. C. Hsueh, and W. Ku, Phys. Rev. B 82, 081106 (2010).

${ }^{21}$ E. D. Isaacs, P. M. Platzman, P. Metcalf, and J. M. Honig, Phys. Rev. Lett. 76, 4211 (1996).

${ }^{22}$ S. Huotari, J. A. Soininen, G. Vankó, G. Monaco, and V. Olevano, Phys. Rev. B 82, 064514 (2010).

${ }^{23}$ N. Vast, L. Reining, V. Olevano, P. Schattschneider, and B. Jouffrey, Phys. Rev. Lett. 88, 037601 (2002).

${ }^{24}$ I. G. Gurtubay, W. Ku, J. M. Pitarke, A. G. Eguiluz, B. C. Larson, J. Tischler, and P. Zschack, Phys. Rev. B 70, 201201(R) (2004).

${ }^{25}$ A. Georges, G. Kotliar, W. Krauth, and M. J. Rozenberg, Rev. Mod. Phys. 68, 13 (1996).

${ }^{26}$ L. Hedin, Phys. Rev. 139, A796 (1965).

${ }^{27}$ D. N. Basov, R. D. Averitt, D. van der Marel, M. Dressel, and K. Haule, Rev. Mod. Phys. 83, 471 (2011).

${ }^{28}$ N. Lin, E. Gull, and A. J. Millis, Phys. Rev. B 80, 161105 (2009); M. I. Katsnelson and A. I. Lichtenstein, J. Phys.: Condens. Matter 22, 382201 (2010).

${ }^{29}$ G. Onida, L. Reining, and A. Rubio, Rev. Mod. Phys. 74, 601 (2002).

${ }^{30}$ E. Runge and E. K. U. Gross, Phys. Rev. Lett. 52, 997 (1984).

${ }^{31}$ S. Botti, A. Schindlmayr, R. Del Sole, and L. Reining, Rep. Prog. Phys. 70, 357 (2007).

${ }^{32}$ Yu. V. Shvyd'ko, J. P. Hill, C. A. Burns, D. S. Coburn et al., J. Electron Spectrosc. Relat. Phenom. (2012), doi: 10.1016/j.elspec.2012.09.003.
${ }^{33}$ S. Huotari, G. Vanko, F. Albergamo, C. Ponchut, H. Graafsma, C. Henriquet, R. Verbeni, and G. Monaco, J. Synchrotron Radiat. 12, 467 (2005)

${ }^{34}$ M. Petersilka, U. J. Gossmann, and E. K. U. Gross, Phys. Rev. Lett. 76, 1212 (1996).

${ }^{35}$ E. K. U. Gross, J. F. Dobson, and M. Petersilka, in Density Functional Theory, edited by R. F. Nalewajski (Springer-Verlag, Berlin, 1996).

${ }^{36}$ P. Romaniello, S. Guyot, and L. Reining, J. Chem. Phys. 131, 154111 (2009).

${ }^{37}$ G. Onida, L. Reining, R. W. Godby, R. Del Sole, and W. Andreoni, Phys. Rev. Lett. 75, 818 (1995).

${ }^{38}$ I. Vasiliev, S. Öğüt, and J. R. Chelikowsky, Phys. Rev. Lett. 82, 1919 (1999); J.-O. Joswig, L. O. Tunturivuori, and R. M. Nieminen, J. Chem. Phys. 128, 014707 (2008).

${ }^{39}$ C. Delerue, M. Lannoo, and G. Allan, Phys. Rev. Lett. 84, 2457 (2000).

${ }^{40}$ A. G. Marinopoulos, L. Reining, A. Rubio, and N. Vast, Phys. Rev. Lett. 91, 046402 (2003).

${ }^{41}$ C. D. Spataru, S. Ismail-Beigi, L. X. Benedict, and S. G. Louie, Appl. Phys. A 78, 1129 (2004); E. Chang, G. Bussi, A. Ruini, and E. Molinari, Phys. Rev. Lett. 92, 196401 (2004).

${ }^{42}$ V. Olevano and L. Reining, Phys. Rev. Lett. 86, 5962 (2001).

${ }^{43}$ In this definition the RPA is not equivalent to the time-dependent Hartree approximation, because $\chi_{K S}$ contains exchange-correlation effects from the Kohn-Sham potential; this will be important later.

${ }^{44}$ Here we do not display spin explicitly.

${ }^{45}$ Here we do not distinguish explicitly between causal and timeordered frameworks, which has of course to be done in practice.

${ }^{46} \mathrm{~A}$ similar behavior has been measured for the paramagnetic metal to antiferromagnetic insulator transition for a momentum transfer $\mathbf{Q}=$ $3.4 \AA^{-1}$ in A. V. Kozhevnikov, M. C. Troparevsky, T. C. Schulthess, A. G. Eguiluz, T. Pylkkänen, L. Paolasini, P. A. Metcalf, G. Monaco, and S. Huotari (unpublished).

${ }^{47}$ For experimental spectra on a wide moment and energy range in the metallic phase see Ref. 48.

${ }^{48}$ R. Verbeni, T. Pylkkänen, S. Huotari, L. Simonelli, G. Vankó, K. Martel, Ch. Henriquet, and G. Monaco, J. Synchrotron Radiat. 16, 469 (2009).

${ }^{49} \mathrm{We}$ have used the experimental lattice parameters for pure PM $\mathrm{V}_{2} \mathrm{O}_{3}$ and for Cr-doped PI $\left(\mathrm{V}_{0.962} \mathrm{Cr}_{0.038}\right)_{2} \mathrm{O}_{3}$ from Ref. 50. LDA ground-state calculations have been performed with the ABINIT code (Ref. 51) following Ref. 13. The spectra have been obtained using the DP code (Ref. 52) with a $6 \times 6 \times 6$ grid of $\mathbf{k}$ points and including 150 bands. The dimension of the microscopic dielectric matrix $\epsilon$ is 509 G vectors.

${ }^{50}$ P. D. Dernier, J. Phys. Chem. Solids 31, 2569 (1970).

${ }^{51}$ X. Gonze, G.-M. Rignanese, M. Verstraete, J.-M. Beuken, Y. Pouillon, R. Caracas, F. Jollet, M. Torrent, G. Zerah, M. Mikami, Ph. Ghosez, M. Veithen, J.-Y. Raty, V. Olevano, F. Bruneval, L. Reining, R. Godby, G. Onida, D. R. Hamann, and D. C. Allan, Z. Kristallogr. 220, 558 (2005).

${ }^{52}$ The DP code is developed by the French node of the ETSF; see: http://www.dp-code.org.

${ }^{53}$ C. Rödl, F. Fuchs, J. Furthmüller, and F. Bechstedt, Phys. Rev. B 79, 235114 (2009).

${ }^{54}$ XAS experiments supported by calculation have tackled this aspect for the high-energy photon threshold; see Ref. 55. 
${ }^{55}$ P. Hansmann, M. W. Haverkort, A. Toschi, G. Sangiovanni, F. Rodolakis, J. P. Rueff, M. Marsi, and K. Held, Phys. Rev. B 85, 115136 (2012).

${ }^{56}$ H.-Ch. Weissker, J. Serrano, S. Huotari, E. Luppi, M. Cazzaniga, F. Bruneval, F. Sottile, G. Monaco, V. Olevano, and L. Reining, Phys. Rev. B 81, 085104 (2010).
${ }^{57}$ S. Botti, N. Vast, L. Reining, V. Olevano, and L. C. Andreani, Phys. Rev. Lett. 89, 216803 (2002).

${ }^{58}$ P. A. Montano, D. L. Price, A. T. Macrander, and B. R. Cooper, Phys. Rev. B 66, 165218 (2002).

${ }^{59}$ See, e.g., M. Gatti, F. Bruneval, V. Olevano, and L. Reining, Phys. Rev. Lett. 99, 266402 (2007). 